\title{
DYNAMICS OF HOROSPHERICAL FLOWS
}

\author{
BY S. G. DANI
}

Let $G$ be a reductive Lie group; that is, the adjoint representation is completely reducible. A subgroup $U$ of $G$ is said to be horospherical if there exists $g \in G$ such that

$$
U=\left\{u \in G \mid g^{j} u g^{-j} \rightarrow e \text { as } j \rightarrow \infty\right\}
$$

where $e$ is the identity element of $G$. The action of a horospherical subgroup (respectively a maximal horospherical subgroup) on a homogeneous space $G / \Gamma$, where $\Gamma$ is a discrete subgroup, is called a horospherical flow (resp. a maximal horospherical flow). The classical horocycle flows associated to surfaces of constant negative curvature, studied by G. A. Hedlund and other authors, can be viewed as horospherical flows on homogeneous spaces of $\operatorname{SL}(2, \mathbf{R})$.

In the case when $\Gamma$ is a co-compact discrete subgroup of $\operatorname{SL}(2, \mathbf{R})$ it was proved by $\mathrm{H}$. Furstenberg that the horocycle flow is uniquely ergodic; i.e. it admits a unique invariant probability measure (cf. [6]). In particular, this implies a result of G. A. Hedlund that the horocycle flow is minimal; i.e. every orbit is dense. Satisfactory generalisations of these assertions for all horospherical flows on compact homogeneous spaces are available in literature (cf. [5] and [7]).

The purpose of this note is to announce similar results for maximal horospherical flows on homogeneous spaces $G / \Gamma$ where $\Gamma$ is any lattice; i.e. $\Gamma$ is a discrete subgroup such that $G / \Gamma$ admits a finite $G$-invariant measure, but may not necessarily be compact. When $G / \Gamma$, as above, is noncompact the horospherical flow fails to be uniquely ergodic. The general task therefore is to obtain a description of all ergodic invariant measures of the horospherical flow. We prove the following.

1. THEOREM. Let $G$ be a reductive Lie group and $\Gamma$ be a lattice in $G$. Let $N$ be a maximal horospherical subgroup of $G$. Let $\sigma$ be an $N$-invariant ergodic probability measure. Then there exists a connected Lie subgroup $L$ of $G$ and an element $x \in G$ such that (i) $L x \Gamma / \Gamma$ is a closed L-orbit in $G / \Gamma$ and (ii) $\sigma$ is $L$ invariant and $\sigma(G / \Gamma-L x \Gamma / \Gamma)=0$.

It is not difficult to show that in the special case when $G / \Gamma$ is compact Theorem 1 yields the known results for maximal horospherical flows.

The proof is achieved using a characterisation of the haar measure of a

Received by the editors April 22, 1980.

1980 Mathematics Subject Classification. Primary 58F11; Secondary 22D40, 28D99, $54 \mathrm{H} 20$. 
semisimple Lie group, proved by the author in [1]. Detailed proofs may be found in [3]. Here we shall describe the candidate for $L$ in a certain (crucial) special case. Let $G$ be a semisimple $\mathbf{R}$-algebraic group defined over $\mathbf{Q}$, admitting no nontrivial compact factors and let $\Gamma$ be a lattice which is commensurable with $G_{Z}$; i.e. $\Gamma \cap G_{Z}$ has finite index in both $\Gamma$ and $G_{Z}$. Let $P$ be a minimal $Q$ parabolic R-subgroup of $G$ and let $R$ be a minimal R-parabolic subgroup of $G$ contained in $P$. Let $N$ be the unipotent radical of $R$. Then $N$ is a maximal horospherical subgroup of $G$. Now let $\sigma$ be a $N$-invariant ergodic probability measure on $G / \Gamma$. Let $\pi$ be the measure on $G$ defined by

$$
\pi(E)=\int_{G / \Gamma} \sum_{\gamma \in \Gamma} \check{\chi}_{E}(g \gamma) d \sigma(g)
$$

where $E$ is any Borel subset of $G$ and $\check{\chi}_{E}$ is the function defined by $\check{\chi}_{E}(g)=1$ or 0 according as $g^{-1} \in E$ or $g^{-1} \notin E$. We recall that by Bruhat decomposition $G$ is a union of finitely many double cosets of the form $P g R, g \in G$. Therefore there exists a double coset $P g_{0} R$ such that (i) $\pi\left(q P g_{0} R\right)>0$ for some $q \in G_{\mathrm{Q}}$ and (ii) $\left.\pi\left(\overline{\left(P g_{0} R\right.}-P g_{0} R\right)\right)=0$ for all $r \in G_{Q}$ (bar overhead stands for closure operation). Let $Q=\left\{g \in G \mid \overline{g P g_{0} R}=\overline{P g_{0} R}\right\}$. Then $Q$ is a parabolic subgroup of $G$. It turns out (a posteriori) that $Q$ is defined over $Q$. Let $V$ be the smallest normal subgroup of $Q$ containing $N$ and let $L=(\overline{V(Q \cap \Gamma)})^{0}$ (the connected component of the identity in $\overline{V(Q \cap \Gamma)}$ ). We prove that assertions (i) and (ii) are satisfied with respect to this subgroup for a suitable $x$.

The general result is then deduced using Margulis's arithmeticity theorem and other well-known results on lattices.

REMARK. It may be noted that if we start with a parabolic subgroup $Q$ which is defined over $\mathbf{Q}$ and contains a maximal horospherical subgroup $N$ and define $L$ as in the preceding paragraph, then there exist closed $L$-orbits on $G / \Gamma$ (as above) which admit a finite $L$-invariant measure. Further the latter is ergodic as a $N$-invariant measure. The assertion in Theorem 1 is indeed the converse of this.

REMARK. For arithmetic lattices the assumption in Theorem 1 that $\sigma$ be a probability measure is redundant. This is because by Theorem 4.1 in [2] finiteness of $\sigma$ is automatic if we just assume $\sigma$ to be locally finite.

Applying Theorem 1 we deduce the following results about subsystems of maximal horospherical flows.

2. Theorem. Let $G, \Gamma$ and $N$ be as in Theorem 1. Then any minimal compact (nonempty) $N$-invariant subset of $G / \Gamma$ is an orbit of a connected Lie subgroup (containing $N$ ).

3. THEOREM. Let $\mathbf{G}$ be a reductive $\mathbf{R}$-algebraic group defined over $\mathbf{Q}$ and let $\Gamma$ be a subgroup commensurable with $G_{\mathrm{Z}}$. Let $P$ be a minimal $Q$-parabolic 
R-subgroup of $G$ and let $N$ be the unipotent radical of a minimal R-parabolic subgroup contained in $P$. Let $V$ be the smallest normal subgroup of $P$ containing $N$

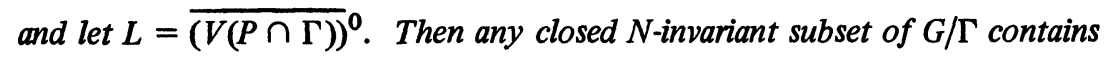
a closed orbit of $L$.

The proof of Theorem 3 depends on the fact that $\Gamma$ being an arithmetic lattice, any closed $N$-invariant nonempty subset of $G / \Gamma$ contains the support of a $N$-invariant measure (cf. Proposition 7.1, [2]).

It may be mentioned here that for certain horospherical flows on $S L(n, \mathbf{R}) / S L(n, \mathrm{Z})$ the closure of any orbit is the orbit of a connected Lie subgroup of $S L(n, \mathbf{R})$. This is proved in [4] by a different method. A similar result is expected to be true in greater generality.

\section{REFERENCES}

1. S. G. Dani, Invariant measures of horospherical flows on noncompact homogeneous spaces, Invent. Math. 47 (1978), 101-138.

2. Invariant measures, minimal sets and a lemma of Margulis, Invent. Math. 51 (1979), 239-260.

3. - Invariant measures and minimal sets of horospherical flows (preprint).

4. S. G. Dani and S. Raghavan, Orbits of euclidean frames under discrete linear groups, Israel J. Math. (to appear).

5. R. Ellis and W. Perrizo, Unique ergodicity of flows on homogeneous spaces, Israel. J. Math. 29 (1978), 276-284.

6. H. Furstenberg, The unique ergodicity of the horocycle flow, Recent Advances in Topological Dynamics (Proc. Conf. Topological Dynamics, Yale Univ., New Haven, Conn., 1972; in honor of Gustov Arnold Hedlund), Lecture Notes in Math., Vol. 318, SpringerVerlag, Berlin and New York, 1973, pp. 95-115. 827-859.

7. W. A. Veech, Unique ergodicity of horospherical flows, Amer. J. Math. 99 (1977),

SCHOOL OF MATHEMATICS, TATA INSTITUTE OF FUNDAMENTAL RESEARCH, HOMI BHABHA ROAD, BOMBAY 400005 INDIA 
